\title{
correspondence
}

\section{A Word from OLAW: COVID-19 guidance}

\section{To the Editor - The NIH Office of} Laboratory Animal Welfare (OLAW) is deeply concerned about the impact of the COVID-19 pandemic on the ability of Assured institutions to support the well-being of animals and personnel during this public health emergency. In Guide Notice NOT-OD-20-088, we provide information on flexibilities in the PHS Policy on Humane Care and Use of Laboratory Animals (Policy) that are applicable to implementing a pandemic plan for animal care and use programs. In this Notice, you will find measures that institutions may implement for conducting semiannual animal facility inspections and IACUC business, plus how to request a waiver for requirements of the PHS Policy.

OLAW's special webinar, Pandemic Contingency Planning and Its Impact on Animal Care, is available online at: https://olaw.nih.gov/education/educationalresources/webinar-2020-03-19.htm.

You'll also find Resources and Supporting Materials including:
- NIH Division of Veterinary Resources Continuity of Services Plans

- Johns Hopkins University COVID-19 Phased Contingency Plan

- Michigan State University's Sample Policy for Conducting IACUC Business in the Event of a Pandemic

- NIH 2008 Pandemic Flu Readiness Plan

- Slide Handouts and webinar transcript

- Slides, with helpful links.

We received many questions prior to the webinar and were unable to address them all within the webinar. We have amended questions and answers to the end of the transcript.

Additional guidance for preparing for a pandemic can be found on the OLAW website in the Frequently Asked Questions section, under Institutional Responsibilities, FAQ G9. If OLAW determines that additional measures must be implemented to meet the needs of the research community and funding components, additional guidance will be announced.
During these uncharted times, OLAW remains committed to working with our community to maintain human and animal health and well-being. The COVID-19 pandemic is a rapidly developing situation. Rest assured that OLAW is monitoring the situation closely. We will continue to issue updated guidance if the situation becomes sufficiently severe and long-lasting that additional guidance is needed. OLAW's dedicated webpage to COVID-19 is https:// olaw.nih.gov/covid-19.htm. Here you find example pandemic plans, webinars, FAQs, and additional resources related to maintaining animal care and use programs during the COVID-19 pandemic.

For more information on NIH's overall response to COVID-19, see the NIH Extramural COVID-19 Response webpage.

\section{Patricia Brown}

Director, OLAW, OER, OD, NIH, HHS, Bethesda, USA. e-mail:brownp@od.nih.gov

Published online: 6 April 2020

https://doi.org/10.1038/s41684-020-0526-0 